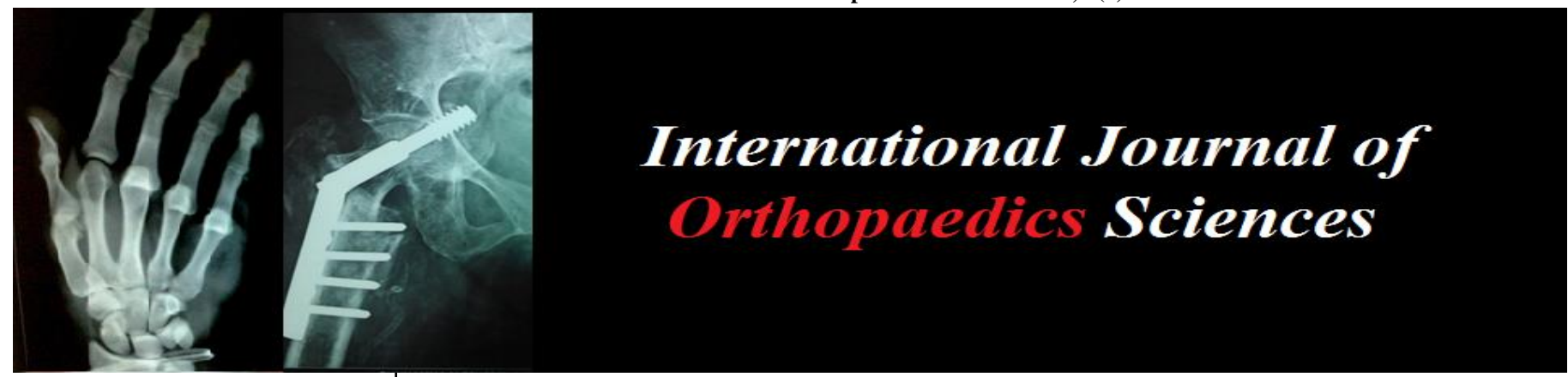

E-ISSN: 2395-1958

P-ISSN: 2706-6630

IJOS 2020; 6(2): 43-49

(C) 2020 IJOS

www.orthopaper.com

Received: 26-02-2020

Accepted: 27-03-2020

Amit Kumar C Jain

Professor,

Department of Surgery,

Rajarajeswari Medical College,

Bengaluru, Karnataka, India

Gopal S

Associate Professor,

Department Of Surgery,

Rajarajeswari Medical College,

Bengaluru, Karnataka, India

\section{Hariprasad TR}

Professor,

Department of Surgery,

Rajarajeswari Medical College, Bengaluru, Karnataka, India

Corresponding Author:

Gopal S

Associate Professor,

Department of Surgery,

Rajarajeswari Medical College,

Bengaluru, Karnataka, India

\section{To determine the acute stump complications in major amputation occurring in hospitalized diabetic foot patients through Amit Jain's typing and scoring system: A prospective study}

\author{
Amit Kumar C Jain, Gopal S and Hariprasad TR
}

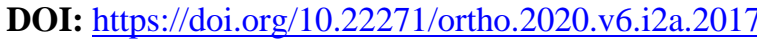

Abstract

Aim: To study the stump complications occurring in patients with major amputations in diabetic foot and analyze them through Amit Jain's classification for diabetic foot complication and Amit Jain's scoring system.

Methods and Material: A prospective study was conducted in Department of surgery of Rajarajeswari medical college, Bengaluru, India. The study period was from June 2018 to may 2019. An IEC approval was obtained for this study.

Results: A total of 15 patients who underwent major amputation were included in the study and majority of them were males. Around $86.7 \%$ of the patients were above 50 years of age. $60 \%$ of the major amputation were done in type 1 diabetic foot complication. $80 \%$ of patients underwent below knee amputation.73.4\% of the patients with major amputation had score of 16 and above. $66.7 \%$ of the patients had some form of stump complication after major amputation and they occurred significantly in patients who had score of 16 and above. Around $66.7 \%$ of patients had their stump closed after major amputations and $80 \%$ of them were significantly done in type 1 diabetic foot complications.

Conclusion: In this study that utilizes Amit Jain's universal classification and scoring system, type 1 diabetic foot complications were the commonest cause for major amputation. Below knee amputation was the most common type of major amputation. Majority of the patients developed some form of stump complication after major amputation. Most major amputations were done in patients who had a score of 16 and above and the stump complications was statistically significant in patients whose score was 16 and above.

Keywords: Diabetes, foot, amputation, stump, infection, scoring, Amit Jain, classification, universal

\section{Introduction}

Amputation, which is a debilitating procedure, was believed to be first performed by the Hammurabi tribe in Babylon as a form of punishment and it was later implemented by Hippocrates as a surgical procedure to save life, more than 2500 years ago ${ }^{[1,2]}$. Today, with growing incidence of diabetes worldwide, the diabetic foot remains one of the common causes of lower limb amputation. In fact, patients with diabetes are 10 times at a higher risk of amputations than non-diabetics ${ }^{[3]}$.

Around $40-70 \%$ pf all the lower extremity amputations are attributed to diabetes mellitus ${ }^{[4]}$. Often, amputation of lower limb affect's one's ability to walk along with impairment in quality of life ${ }^{[5]}$. In amputations for diabetic foot complications, it is often preferred to be a minor amputation over major amputation ${ }^{[6]}$. Amputation performed at or below ankle joint are considered to be minor amputations whereas amputation above ankle joint are major amputations $[1,6,7]$.

Major amputations are often associated with increased morbidity and mortality compared to minor amputation ${ }^{[6,8,9]}$. One such problem with major amputation is stump complication.

This study was aimed to analyze and study the stump complications occurring in major amputation in diabetic foot patients and to distribute it through Amit Jain's typing and scoring system [9, 10, 11, 12]. Amit Jain's universal classification is a simple, descriptive 3 tier classification that divides diabetic foot complications into 3 simple types which encompasses 
most of the common lesion seen in diabetic foot worldwide [Figure 1] and Amit Jain's surgical scoring system is a new scoring system [Table 1] for diabetic foot complication that predicts the risk of major amputation and both were proposed from Indian subcontinent.

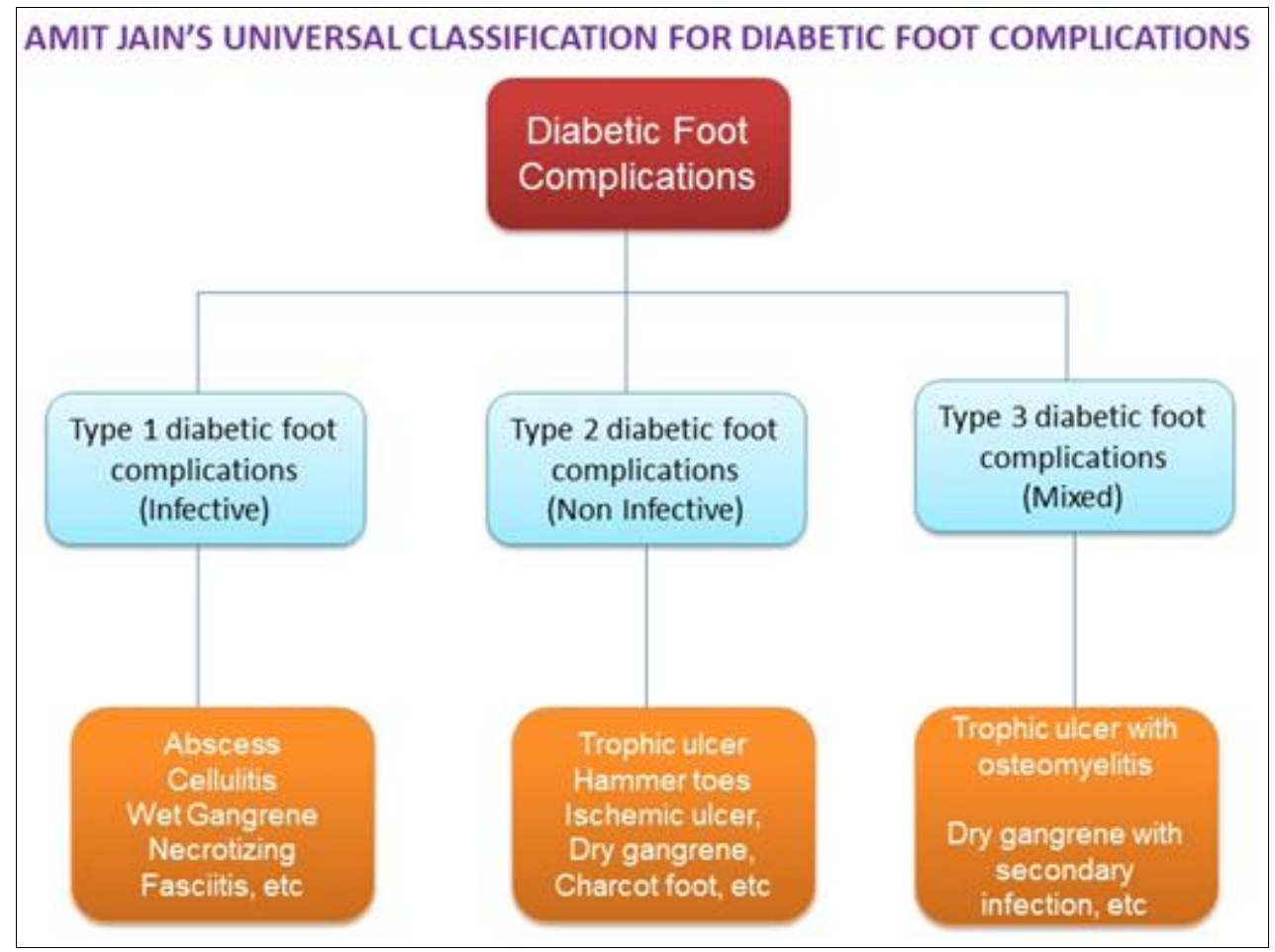

Fig 1: Showing Amit Jain's universal classification for diabetic foot complications

Table 1: Showing Amit Jain's scoring system for diabetic foot

\begin{tabular}{|c|c|c|c|c|c|}
\hline S. No & Characteristics & \multicolumn{4}{|c|}{ Involvement of Foot } \\
\hline 1. & Presence of Ulcer & No Ulcer $\rightarrow 0$ & Forefoot Ulcer $\rightarrow 2$ & Midfoot Ulcer $\rightarrow 4$ & $\begin{array}{l}\text { Hindfoot Ulcer/ Full } \\
\text { Foot/Beyound } \rightarrow 6\end{array}$ \\
\hline 2. & $\begin{array}{l}\text { Osteomyelitis } \\
\text { [O.M] }\end{array}$ & No O.M $\rightarrow 0$ & Forefoot O.M $\rightarrow 2$ & Midfoot O.M $\rightarrow 4$ & Hindfoot O.M $\rightarrow 6$ \\
\hline 3. & Presence Of Pus & No Pus $\rightarrow 0$ & $\begin{array}{c}\text { Forefoot Pus/Dorsum } \rightarrow \\
2\end{array}$ & Midfoot Pus $\rightarrow 4$ & $\begin{array}{c}\text { Hindfoot Pus/Beyond It } \rightarrow \\
6\end{array}$ \\
\hline 4. & $\begin{array}{l}\text { Gangrene } \\
\text { [Dry/Wet] }\end{array}$ & No Gangrene $\rightarrow 0$ & Forefoot Gangrene $\rightarrow 2$ & Midfoot Gangrene $\rightarrow 4$ & $\begin{array}{c}\text { Hindfoot } \\
\text { Gangrene/Beyond } \rightarrow 8\end{array}$ \\
\hline 5. & $\begin{array}{c}\text { Peripheral } \\
\text { Arterial Disease }\end{array}$ & $\begin{array}{c}\text { No Peripheral } \\
\text { Arterial Disease } \rightarrow 0\end{array}$ & Mild $\rightarrow 2$ & Moderate $\rightarrow 4$ & Severe $\rightarrow 8$ \\
\hline 6. & $\begin{array}{c}\text { Charcot Foot/ } \\
\text { Destroyed Joints }\end{array}$ & No $\rightarrow 0$ & Forefoot $\rightarrow 2$ & Midfoot $\rightarrow 4$ & Hindfoot/Whole Foot $\rightarrow 8$ \\
\hline 7. & Skin Necrosis & $\mathrm{No} \rightarrow 0$ & Forefoot Necrosis $\rightarrow 2$ & Midfoot Necrosis $\rightarrow 4$ & $\begin{array}{c}\text { Hindfoot } \\
\text { Necrosis/Beyond } \rightarrow 8\end{array}$ \\
\hline 8. & $\begin{array}{l}\text { Surrounding } \\
\text { Cellulitis }\end{array}$ & $\mathrm{No} \rightarrow 0$ & Upto Forefoot $\rightarrow 2$ & Upto Midfoot $\rightarrow 4$ & $\begin{array}{c}\text { Upto Hindfoot \& Beyond } \rightarrow \\
6\end{array}$ \\
\hline 9. & Past Amputation & No $\rightarrow 0$ & Toe Amputation $\rightarrow 2$ & $\begin{array}{l}\text { Forefoot Amputation } \rightarrow \\
4\end{array}$ & Midfoot Amputation $\rightarrow 6$ \\
\hline 10. & $\begin{array}{c}\text { Presence Of Gas } \\
\text {-Radiologically }\end{array}$ & No $\rightarrow 0$ & Gas In Forefoot $\rightarrow 1$ & Gas In/Upto Midfoot $\rightarrow 2$ & Gas In/Upto Hindfoot $\rightarrow 3$ \\
\hline 11. & Myonecrosis & No $\rightarrow 0$ & $\begin{array}{l}\text { Myonecrosis Involving } \\
\text { Single Muscle Group } \rightarrow 2\end{array}$ & $\begin{array}{c}\text { Myonecrosis Involving } \\
\text { More Than One Group } \\
\rightarrow 4 \\
\end{array}$ & $\begin{array}{c}\text { Myonecrosis Of Entire Foot } \\
\text { Muscle With Extension To } \\
\text { Leg } \rightarrow 8\end{array}$ \\
\hline 12. & $\begin{array}{c}\text { Joint } \\
\text { Involvement }\end{array}$ & No $\rightarrow 0$ & $\begin{array}{l}\text { Forefoot Joint } \\
\text { Exposure } \rightarrow 2\end{array}$ & $\begin{array}{l}\text { Midfoot Joint } \\
\text { Exposure } \rightarrow 4\end{array}$ & $\begin{array}{c}\text { Hindfoot Joint Exposure } \rightarrow \\
6\end{array}$ \\
\hline 13. & Septic Shock & \multicolumn{2}{|c|}{ No $\rightarrow 0$} & \multicolumn{2}{|c|}{ Present $\rightarrow 2$} \\
\hline 14. & $\begin{array}{c}\text { Renal Failure } \\
\text { [Acute] }\end{array}$ & \multicolumn{2}{|c|}{ No $\rightarrow 0$} & \multicolumn{2}{|c|}{ Present $\rightarrow 2$} \\
\hline 15. & Smoking & \multicolumn{2}{|c|}{ No $\rightarrow 0$} & \multicolumn{2}{|c|}{ Present $\rightarrow 2$} \\
\hline 16. & Surgeon Factor & \multicolumn{2}{|c|}{ Podiatric/Diabetic Foot Surgeon $\rightarrow 0$} & \multicolumn{2}{|c|}{ Other Surgeons $\rightarrow 2$} \\
\hline
\end{tabular}

\section{Methods and Material}

A prospective descriptive study was done in department of surgery at Rajarajeswari medical college, Bengaluru, India. It is a tertiary care teaching hospital that mainly caters patients from rural area. The study period was from June 2018 to May 2019. An Institutional ethics committee clearance was obtained for this study (RRMCH-IEC/26/2017-18).

The following were the inclusion and exclusion criteria. 


\section{Inclusion Criteria}

1. All the diabetic foot patients admitted and who underwent major amputation in department of surgery were included.

\section{Exclusion Criteria}

1. Patients operated outside

2. Patients operated in other departments

3. Patients discharged against medical advice

\section{Data Analysis $[13,14,15]$}

Data was analyzed using statistical software SPSS 22.0 and R environment ver.3.2.2. Microsoft word and excel were used to generate graphs and tables. Both descriptive and inferential statistics were carried out in the study. Results on continuous measurements were presented on Mean \pm SD (Min-Max) and results on categorical measurements were presented in number (\%). Significance was assessed at 5\% level of significance.

The following assumption on data Is made

- Dependent variables should be normally distributed,

- Samples drawn from the population should be random

- Cases of the samples should be independent

Student $t$ test (two tailed, independent) has been used to find the significance of study parameters on continuous scale between two groups (Inter group analysis) on metric parameters. Chi-square/ Fisher Exact test has been used to find the significance of study parameters on categorical scale between two or more groups, Non-parametric setting for Qualitative data analysis. Fisher exact test was used when samples were very small.

\section{Significant Figures}

+ Suggestive significance $(\mathrm{P}$ value: $0.05<\mathrm{P}<0.10)$

* Moderately significant $(\mathrm{P}$ value: $0.01<\mathrm{P} 0.05$ )

** Strongly significant ( $\mathrm{P}$ value: $\mathrm{P} \leq 0.01)$.

\section{Results}

A total of 15 patients were included in this study. 13 patients $(86.75 \%)$ were males and 2 patients $(13.3 \%)$ were females [Figure 2].

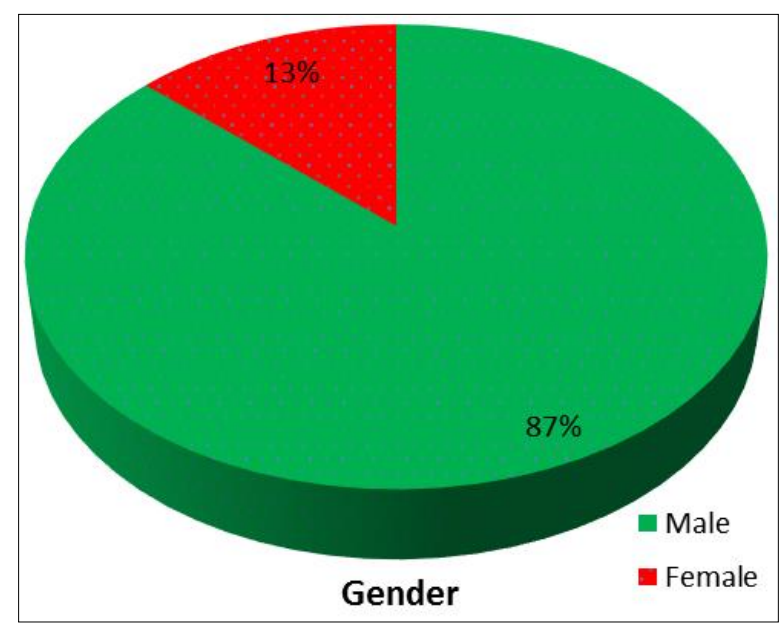

Fig 2: Showing gender distribution

$86.7 \%$ of the patients were above 50 years of age [Table 2] and $66.65 \%$ of them had diabetes of less than 12 years [Table 3].
Table 2: Showing age distribution of patients studied

\begin{tabular}{|c|c|}
\hline Age in years & Percentage \% \\
\hline$<50$ & 13.3 \\
\hline $50-60$ & 46.7 \\
\hline $61-70$ & 40 \\
\hline Total & 100 \\
\hline
\end{tabular}

Table 3: Showing Diabetes mellitus duration distribution of patients studied

\begin{tabular}{|c|c|}
\hline Diabetes Duration & Percentage \% \\
\hline$<6$ & 33.3 \\
\hline $6-12$ & 33.3 \\
\hline $12-24$ & 26.7 \\
\hline$>24$ & 6.7 \\
\hline Total & 100 \\
\hline
\end{tabular}

9 patients (60\%) had type 1 diabetic foot complications and 6 patients (40\%) had type 3 diabetic foot complication [Table 4]. Abscess, necrotizing fasciitis and wet gangrene which are type 1 diabetic foot complications occurred in equal percentage.

Table 4: Showing type of diabetic foot complication and pathological lesion as per Amit Jain's universal classification for diabetic foot complication

\begin{tabular}{|c|c|c|}
\hline $\begin{array}{c}\text { Type of diabetic foot } \\
\text { complication }\end{array}$ & Pathology & $\begin{array}{c}\text { Percentage } \\
\text { \% }\end{array}$ \\
\hline \multirow{4}{*}{$\begin{array}{c}\text { Type 1 diabetic foot } \\
\text { complication }\end{array}$} & Abscess & 20 \\
\cline { 2 - 3 } & $\begin{array}{c}\text { Necrotizing } \\
\text { fasciitis }\end{array}$ & 20 \\
\cline { 2 - 3 } & Wet gangrene & 20 \\
\hline \multirow{4}{*}{$\begin{array}{c}\text { Type 3 diabetic foot } \\
\text { complication }\end{array}$} & $\begin{array}{c}\text { Infected ulcer } \\
\text { fonfect Charcot }\end{array}$ & 20 \\
\cline { 2 - 3 } & $\begin{array}{c}\text { Infected dry } \\
\text { gangrene }\end{array}$ & 6.7 \\
\cline { 2 - 3 } & Total & 100 \\
\hline
\end{tabular}

$20 \%$ of the patients who underwent major amputation had underlying osteomyelitis [Figure 3].

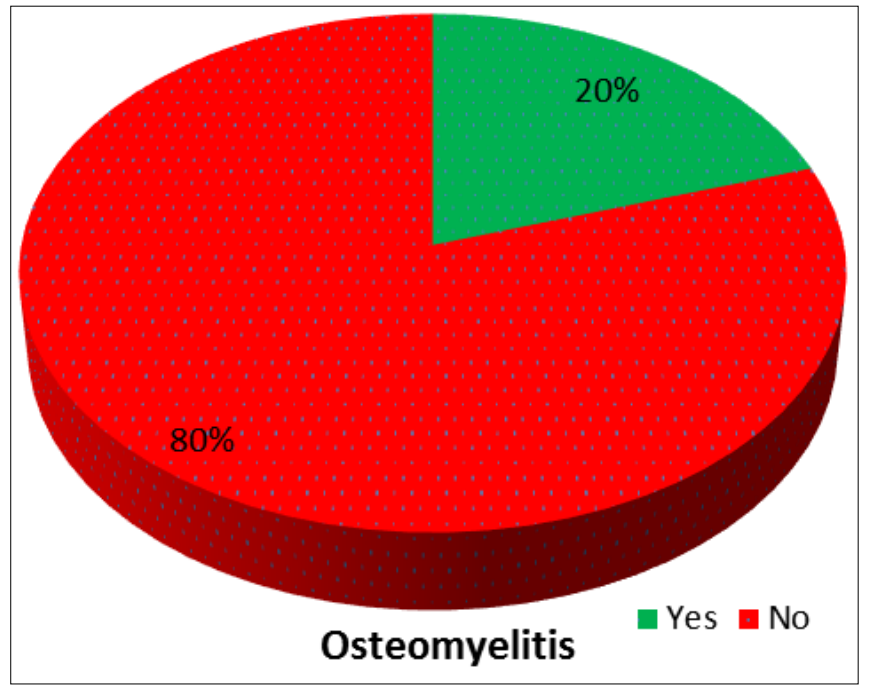

Fig 3: Showing osteomyelitis distribution

Below knee amputation was the most common major amputation $(80 \%)$ in this series with below knee amputation and above knee amputation ration being 4:1 [Figure 4]. 


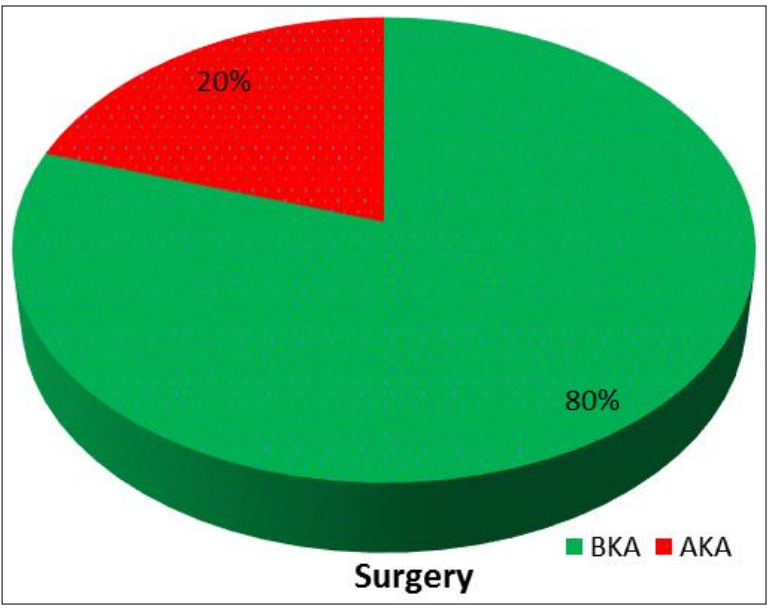

Fig 4: Showing distribution of major amputations

There was no relation between age, gender, diabetes mellitus duration, diagnosis, presence of osteomyelitis, peripheral vascular disease, side of foot involved, resurgeries or stump status with major amputation [Table 5, 6, 7].

Table 5: Showing association of clinical variables in relation to type of surgery of patients studied

\begin{tabular}{|c|c|c|c|c|}
\hline \multirow{3}{*}{ Variables } & \multicolumn{2}{|c|}{ Surgery } & \multirow{3}{*}{$\begin{array}{l}\text { Total } \\
\mathrm{n}=15)\end{array}$} & \multirow{3}{*}{$P$ value } \\
\hline & BKA & AKA & & \\
\hline & $\mathrm{n}=12)$ & $n=3$ ) & & \\
\hline \multicolumn{5}{|l|}{ Age in years } \\
\hline$<50$ & 16.7 & 0 & 13.3 & \multirow{3}{*}{0.723} \\
\hline $50-60$ & 50 & 33.3 & 46.7 & \\
\hline $61-70$ & 33.3 & 66.7 & 40 & \\
\hline \multicolumn{5}{|l|}{ Gender } \\
\hline Male & 83.3 & 100 & 86.7 & \multirow{2}{*}{1} \\
\hline Female & 16.7 & 0 & 13.3 & \\
\hline \multicolumn{5}{|l|}{ Diabetes mellitus duration } \\
\hline$<6$ & 33.3 & 33.3 & 33.3 & \multirow{4}{*}{1} \\
\hline $6-12$ & 33.3 & 33.3 & 33.3 & \\
\hline $12-24$ & 25 & 33.3 & 26.7 & \\
\hline$>24$ & 8.3 & 0 & 6.7 & \\
\hline \multicolumn{5}{|l|}{ Diagnosis } \\
\hline Abscess & 25 & 0 & 20 & \multirow{3}{*}{1} \\
\hline Infected ulcer & 16.7 & 33.3 & 20 & \\
\hline Necrotizing fasciitis & 16.7 & 33.3 & 20 & \\
\hline Wet gangrene & 16.7 & 33.3 & 20 & \\
\hline Infected Charcot foot & 16.7 & 0 & 13.3 & \\
\hline Infected dry gangrene & 8.3 & 0 & 6.7 & \\
\hline
\end{tabular}

Table 6: Showing association of clinical variables in relation to type of surgery of patients studied

\begin{tabular}{|c|c|c|c|c|}
\hline \multirow{3}{*}{ Variables } & \multicolumn{2}{|c|}{ Surgery } & \multirow{3}{*}{$\begin{array}{c}\text { Total } \\
(n=15) \\
\end{array}$} & \multirow{3}{*}{$\begin{array}{c}P \\
\text { value }\end{array}$} \\
\hline & BKA & AKA & & \\
\hline & $\mathrm{n}=12$ ) & $n=3$ ) & & \\
\hline \multicolumn{5}{|l|}{ Osteomyelitis } \\
\hline Yes & 16.7 & 33.3 & 20 & \multirow{2}{*}{0.516} \\
\hline No & 83.3 & 66.7 & 80 & \\
\hline \multicolumn{5}{|l|}{ PVD } \\
\hline Yes & 8.3 & 0 & 6.7 & \multirow{2}{*}{1} \\
\hline No & 91.7 & 100 & 93.3 & \\
\hline \multicolumn{5}{|l|}{ Side Foot } \\
\hline Right & 50 & 33.3 & 46.7 & \multirow[b]{2}{*}{1} \\
\hline Left & 50 & 66.7 & 53.3 & \\
\hline \multicolumn{5}{|l|}{ Stump [Closed /Open] } \\
\hline Open & 41.7 & 0 & 33.3 & \multirow{2}{*}{0.505} \\
\hline Closed & 58.3 & 100 & 66.7 & \\
\hline
\end{tabular}

Table 7: Showing association of Resurgeries in relation to type of surgery of patients studied

\begin{tabular}{|c|c|c|c|c|}
\hline \multirow{2}{*}{ Resurgery } & Surgery & & Total & \multirow{2}{*}{ P Value } \\
\cline { 2 - 4 } & BKA & AKA & & \\
\hline Yes & 66.7 & 66.7 & 66.7 & \multirow{2}{*}{1.00} \\
\hline No & 33.3 & 33.3 & 33.3 & \\
\hline Total & 100 & 100 & 100 & \\
\hline
\end{tabular}

There was also no relation of type of diabetic foot complication, risk categorization, hypertension, chronic kidney disease, ischemic heart disease, history of past amputation, stump complication, type of stump complication with major amputation [Table 8 and 9]. $73.4 \%$ of the patients with major amputation had a score of 16 and above [Figure 5]

Table 8: Association of clinical variables in relation to type of surgery of patients studied

\begin{tabular}{|c|c|c|c|c|}
\hline \multirow{3}{*}{ Variables } & \multicolumn{2}{|c|}{ Surgery } & \multirow{3}{*}{$\begin{array}{r}\text { Total } \\
\mathbf{n}=15)\end{array}$} & \multirow{3}{*}{$\begin{array}{c}\mathbf{P} \\
\text { value }\end{array}$} \\
\hline & BKA & AKA & & \\
\hline & $\mathrm{n}=12)$ & $n=3$ ) & & \\
\hline \multicolumn{5}{|l|}{$\begin{array}{c}\text { Type Diabetic Foot } \\
\text { Complications }\end{array}$} \\
\hline Type I & 58.3 & 66.7 & 60 & \multirow{3}{*}{1} \\
\hline Type II & 0 & 0 & 0 & \\
\hline Type III & 41.7 & 33.3 & 40 & \\
\hline \multicolumn{5}{|l|}{ Amit Jain's Scoring } \\
\hline$<15$ & 33.3 & 0 & 26.7 & \multirow{2}{*}{0.516} \\
\hline$>15$ & 66.7 & 100 & 73.3 & \\
\hline \multicolumn{5}{|l|}{ Risk Category } \\
\hline No risk $(0-5)$ & 0 & 0 & 0 & \multirow{6}{*}{0.859} \\
\hline Low risk (6-10) & 0 & 0 & 0 & \\
\hline Moderate (11-15) & 33.3 & 0 & 26.7 & \\
\hline High (16-20) & 16.7 & 33.3 & 20 & \\
\hline Very high (21-25) & 25 & 33.3 & 26.7 & \\
\hline Inevitable ( $26 \&$ above $)$ & 25 & 33.3 & 26.7 & \\
\hline \multicolumn{5}{|l|}{ Hypertension } \\
\hline Yes & 33.3 & 33.3 & 33.3 & \multirow{2}{*}{1} \\
\hline No & 66.7 & 66.7 & 66.7 & \\
\hline \multicolumn{5}{|l|}{ CKD } \\
\hline Yes & 16.7 & 33.3 & 20 & \multirow{2}{*}{0.516} \\
\hline No & 83.3 & 66.7 & 80 & \\
\hline \multicolumn{5}{|l|}{ IHD } \\
\hline Yes & 0 & 0 & 0 & \multirow{2}{*}{1} \\
\hline No & 100 & 100 & 100 & \\
\hline
\end{tabular}

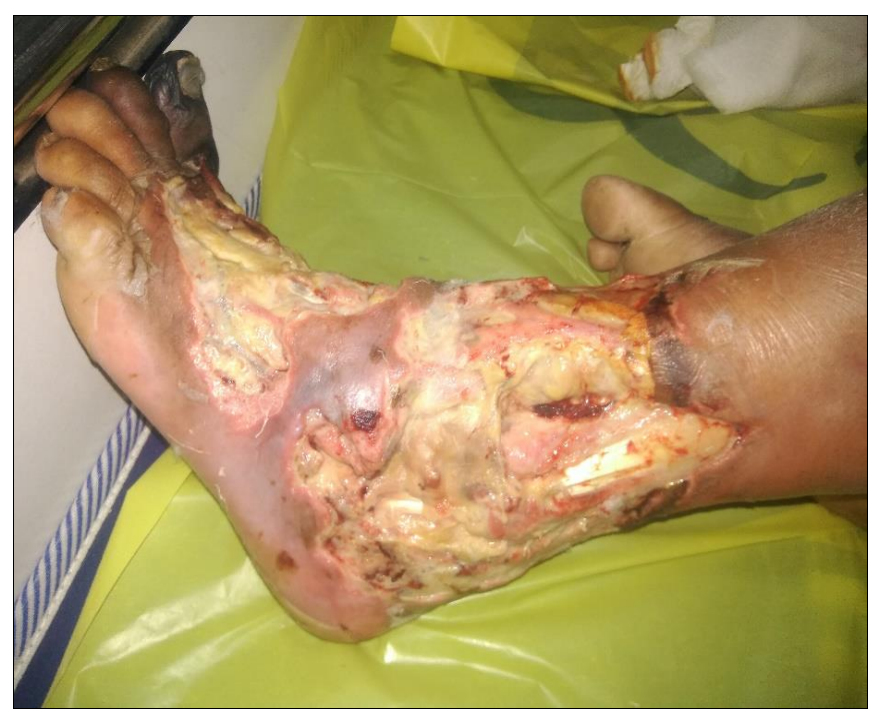

Fig 5: Showing patient who ended in below knee amputation. He was operated at another hospital for abscess foot. His final score was

16 [Ulcer $6+$ Gangrene $2+$ Pus $6+$ Surgeon factor 2] and he belonged to high risk category. 
Table 9: Showing association of clinical variables in relation to type of surgery of patients studied

\begin{tabular}{|c|c|c|c|c|}
\hline \multirow{3}{*}{ Variables } & \multicolumn{2}{|c|}{ Surgery } & \multirow{3}{*}{$\begin{array}{l}\text { Total } \\
n=15)\end{array}$} & \multirow{3}{*}{$P$ value } \\
\hline & BKA & AKA & & \\
\hline & $\mathrm{n}=12)$ & $\mathbf{n}=\mathbf{3})$ & & \\
\hline \multicolumn{5}{|l|}{ Past Amputation } \\
\hline Yes & 25 & 33.3 & 26.7 & \multirow{2}{*}{1} \\
\hline No & 75 & 66.7 & 73.3 & \\
\hline \multicolumn{5}{|l|}{ Stump Complications } \\
\hline Yes & 66.7 & 66.7 & 66.7 & \multirow{2}{*}{1} \\
\hline No & 33.3 & 33.3 & 33.3 & \\
\hline \multicolumn{5}{|l|}{ Stump Type } \\
\hline Abscess & 58.3 & 33.3 & 53.3 & \multirow{5}{*}{0.525} \\
\hline Flap necrosis & 0 & 33.3 & 6.7 & \\
\hline Wound dehiscence & 8.3 & 0 & 6.7 & \\
\hline Haemorrhage & 8.3 & 0 & 6.7 & \\
\hline No complication & 25 & 33.3 & 26.7 & \\
\hline \multicolumn{5}{|l|}{ Infective/Non-Infective } \\
\hline Infective & 66.7 & 66.7 & 66.7 & \multirow{2}{*}{1} \\
\hline Non infective & 33.3 & 33.3 & 33.3 & \\
\hline
\end{tabular}

There was also no correlation between stump status and type of stump complication with peripheral vascular disease [Table 10].

Table 10: Showing association of clinical variables in relation to peripheral vascular disease of patients studied

\begin{tabular}{|c|c|c|c|c|}
\hline \multirow{3}{*}{ Variables } & \multicolumn{2}{|c|}{ PVD } & \multirow{3}{*}{$\begin{array}{l}\text { Total } \\
n=15)\end{array}$} & \multirow{3}{*}{$P$ value } \\
\hline & Yes & No & & \\
\hline & $\mathrm{n}=1)$ & $\mathrm{n}=14)$ & & \\
\hline \multicolumn{5}{|l|}{ Stump Complications } \\
\hline Yes & 100 & 64.3 & 66.7 & \multirow{2}{*}{1} \\
\hline No & 0 & 35.7 & 33.3 & \\
\hline \multicolumn{5}{|l|}{ Stump [Closed/ Open] } \\
\hline Open & 100 & 28.6 & 33.3 & \multirow{2}{*}{0.333} \\
\hline Closed & 0 & 71.4 & 66.7 & \\
\hline \multicolumn{5}{|l|}{ Stump Type } \\
\hline Abscess & 100 & 50 & 53.3 & \multirow{5}{*}{1} \\
\hline Flap necrosis & 0 & 7.1 & 6.7 & \\
\hline Wound dehiscence & 0 & 7.1 & 6.7 & \\
\hline No complication & 0 & 28.6 & 26.7 & \\
\hline Haemorrhage & 0 & 7.1 & 6.7 & \\
\hline \multicolumn{5}{|l|}{ Infective/Non-Infective } \\
\hline Infective & 100 & 64.3 & 66.7 & \multirow{2}{*}{1} \\
\hline Non infective & 0 & 35.7 & 33.3 & \\
\hline
\end{tabular}

The type of diabetic foot complication was significantly associated with stump status. Around $80 \%$ of type 3 diabetic foot complications had open stump whereas $80 \%$ of type 1 diabetic foot complications had closed stump (P-0.089+). There was no relation of type of diabetic foot complication with type of stump complication. There is also no association between risk categorization, re-surgeries, type of stump complication with stump status [Table 11].

Table 11: Showing association of clinical variables in relation to Stump being Closed/Open of patients studied

\begin{tabular}{|c|c|c|c|c|}
\hline \multirow{3}{*}{ Variables } & \multicolumn{2}{|c|}{$\begin{array}{c}\text { Stump } \\
{[\text { Closed/Open] }}\end{array}$} & \multirow{3}{*}{$\begin{array}{c}\text { Total } \\
(n=15)\end{array}$} & \multirow{3}{*}{$\begin{array}{c}\mathbf{P} \\
\text { value }\end{array}$} \\
\hline & Open & Closed & & \\
\hline & $n=5)$ & $\mathbf{n = 1 0 )}$ & & \\
\hline \multicolumn{5}{|l|}{ Amit Jain's Scoring } \\
\hline$<15$ & 20 & 30 & 26.7 & \multirow{2}{*}{1} \\
\hline$>15$ & 80 & 70 & 73.3 & \\
\hline \multicolumn{5}{|l|}{ Risk Category } \\
\hline No risk & 0 & 0 & 0 & \multirow{6}{*}{1} \\
\hline Low risk & 0 & 0 & 0 & \\
\hline Moderate & 20 & 30 & 26.7 & \\
\hline High & 20 & 20 & 20 & \\
\hline Very high & 40 & 20 & 26.7 & \\
\hline Inevitable & 20 & 30 & 26.7 & \\
\hline \multicolumn{5}{|l|}{$\begin{array}{l}\text { Type Diabetic Foot } \\
\text { Complications }\end{array}$} \\
\hline Type I & 20 & 80 & 60 & \multirow{3}{*}{$0.089+$} \\
\hline Type II & 0 & 0 & 0 & \\
\hline Type III & 80 & 20 & 40 & \\
\hline \multicolumn{5}{|l|}{ Resurgeries } \\
\hline Yes & 100 & 50 & 66.7 & \multirow{2}{*}{0.101} \\
\hline No & 0 & 50 & 33.3 & \\
\hline \multicolumn{5}{|l|}{ Stump Complications } \\
\hline Yes & 80 & 60 & 66.7 & \multirow{2}{*}{0.6} \\
\hline No & 20 & 40 & 33.3 & \\
\hline \multicolumn{5}{|l|}{ Stump Type } \\
\hline Abscess & 100 & 30 & 53.3 & \multirow{5}{*}{0.184} \\
\hline Flap necrosis & 0 & 10 & 6.7 & \\
\hline Wound dehiscence & 0 & 10 & 6.7 & \\
\hline Haemorrhage & 0 & 10 & 6.7 & \\
\hline No complication & 0 & 40 & 26.7 & \\
\hline \multicolumn{5}{|l|}{ Infective/Non-Infective } \\
\hline Infective 1 & 100 & 50 & 66.7 & \multirow{2}{*}{0.101} \\
\hline Non infective & 0 & 50 & 33.3 & \\
\hline
\end{tabular}

All the patients who had score of more than 15 (High risk and above) had significant association [Figure 6] with stump complications (P-0.077+) and they were infective. Although no association existed between score with stump status, type of major amputation, type of diabetic foot complication or resurgeries in this series [Table 12].

Table 12: Showing association of study variables in relation to Amit Jain's scoring of patients studied

\begin{tabular}{|c|c|c|c|c|}
\hline \multirow{3}{*}{ Variables } & \multicolumn{2}{|c|}{ Amit Jain Scoring (>15) } & \multirow{3}{*}{$\begin{array}{l}\text { Total } \\
(n=15)\end{array}$} & \multirow{3}{*}{$P$ value } \\
\hline & Yes & No & & \\
\hline & $(n=4)$ & $\mathrm{n}=11)$ & & \\
\hline \multicolumn{5}{|l|}{ Stump Complications } \\
\hline Yes & 25 & 81.8 & 66.7 & \multirow{2}{*}{$0.077+$} \\
\hline No & 75 & 18.2 & 33.3 & \\
\hline \multicolumn{5}{|l|}{ Stump Type } \\
\hline Abscess & 25 & 63.6 & 53.3 & \multirow{5}{*}{0.292} \\
\hline Flap necrosis & 0 & 9.1 & 6.7 & \\
\hline Wound dehiscence & 0 & 9.1 & 6.7 & \\
\hline No complication & 50 & 18.2 & 26.7 & \\
\hline Haemorrhage & 25 & 0 & 6.7 & \\
\hline \multicolumn{5}{|l|}{ Infective/Non-Infective } \\
\hline Infective & 25 & 81.8 & 66.7 & \multirow{2}{*}{$0.077+$} \\
\hline Non infective & 75 & 18.2 & 33.3 & \\
\hline Stump [Closed /Open] & & & & \\
\hline
\end{tabular}




\begin{tabular}{|c|c|c|c|c|}
\hline Open & 25 & 36.4 & 33.3 & \multirow{2}{*}{1} \\
\hline Closed & 75 & 63.6 & 66.7 & \\
\hline \multicolumn{5}{|c|}{ Type of Diabetic Foot Complications } \\
\hline Type I & 75 & 54.5 & 60 & \multirow{3}{*}{0.604} \\
\hline Type II & 0 & 0 & 0 & \\
\hline Type III & 25 & 45.5 & 40 & \\
\hline \multicolumn{5}{|l|}{ Surgery } \\
\hline BKA & 100 & 72.7 & 80 & \multirow{2}{*}{0.516} \\
\hline AKA & 0 & 27.3 & 20 & \\
\hline \multicolumn{5}{|c|}{ Resurgeries } \\
\hline Yes & 50 & 72.7 & 66.7 & \multirow{2}{*}{0.56} \\
\hline No & 50 & 27.3 & 33.3 & \\
\hline
\end{tabular}

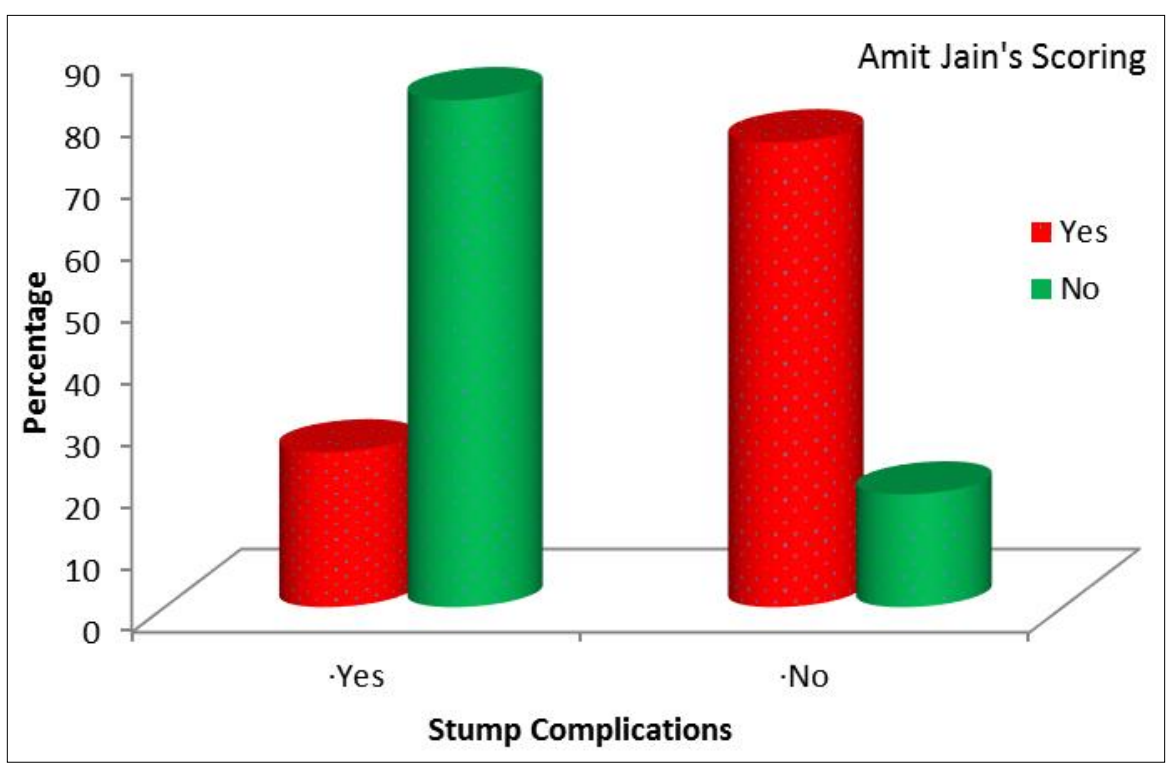

Fig 6: Showing the distribution of the stump complication in relation to Amit Jain's scoring system

\section{Discussion}

Although many consider amputations to be mutilating surgery, there are others who consider it to be a reconstruction as it is a step towards rehabilitation ${ }^{[16]}$. Around $85 \%$ of all amputation were preceded by ulcers and $58 \%$ of the ulcers were likely to get infected ${ }^{[17,18]}$. Diabetic foot infections have 150 -fold increased risk of amputation in lower limb in diabetic patients ${ }^{[18]}$. Recent studies done by Jain et al. found the major amputations in diabetic foot in major teaching hospitals to be between $16 \%$ to $20 \%{ }^{[19,20]}$.

Major amputation in diabetes patient is associated with significant social, economic and psychological impact on patients and their families along with decreased survival [21, ${ }^{22]}$. Further, major amputations have stump complications that increases morbidity, prolonged hospitalization and cost of treatment.

Some of the well-known post-operative complication which are acute in nature are wound infections, wound dehiscence, hematoma, phantom pain. Stump gangrene, flap necrosis, etc [2, 9, 23, 24]. In Chalya et al. series ${ }^{[2]}$, the stump complication was $33.3 \%$ whereas in Ajibade et al. series, it was $31 \%{ }^{[9]}$. In Jain et al. series done exclusively on stump complications [9], it was around $43.7 \%$ wherein $78.57 \%$ of stump complication occurring after major amputation where in type 1 diabetic foot complication with diabetic foot abscess being the commonest cause for which amputation was done.

In this series of stump complication, $60 \%$ of patients who underwent major amputation were operated for type 1 diabetic foot complications with abscess, necrotizing fasciitis and wet gangrene being common in equal proportion with $66.7 \%$ of them having some form of postoperative stump complication. In Chalya et al. series ${ }^{[2]}, 21 \%$ of them had wound infection of stump and $3.1 \%$ had wound dehiscence. In Umaru et al. series, $28.3 \%$ had wound infection and $12.3 \%$ had wound dehiscence ${ }^{[22]}$. In Salawu et al. series ${ }^{[25]}, 10.7 \%$ had wound infection and $6.4 \%$ had flap necrosis after major amputation of extremities. In Jain et al. series [9] that studied stump complications in major amputation, $64.29 \%$ of the patients had wound infection and it was commonest complication among stump complication followed by flap necrosis [14.28\%]. In this series, 53.3\% of stump had wound infection [Figure 7] and $6.7 \%$ had flap necrosis, wound dehiscence and hemorrhage each.

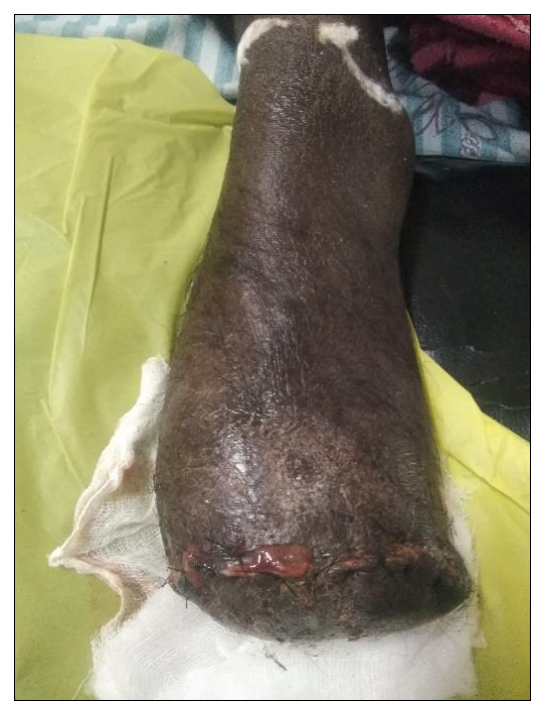

Fig 7: Showing stump infection after below knee amputation. Note the pus discharge. 
In Jain et al. series [9], $85.7 \%$ of all the stump complication had score of 16 and above. Around $35.71 \%$ of them were in high risk category and Amputation inevitable category as per Amit Jain's scoring system. In this series, $66.7 \%$ had stump complications and these complications were statistically significant in patients with score of 16 and above in this series. In Jain et al. series [9], $14.29 \%$ of patient with stump complications had in hospital mortality. In our series, there was no inpatient mortality.

\section{Conclusion}

Major amputation is a distressing procedure with significant morbidity. Below knee amputations are the most common type of major amputations in diabetic foot and type 1 diabetic foot complication accounted for $60 \%$ of major amputation. $66.7 \%$ of patients with major amputation had postoperative stump complication with stump infection being most common. Majority of the type 1 diabetic foot complications had their stump being closed at surgery whereas the patients with type 3 diabetic foot complications had their stump left open and it was statistically significant. Patients with Amit Jain's score of 16 and above had significant stump complications and most of them were of infective type.

\section{Acknowledgement}

The author would like to thank Dr KP Suresh, Scientist (Biostatistics), National Institute of Veterinary Epidemiology and Disease Informatics (NIVEDI), Bangalore, for reviewing the research methodology and statistical results of the study.

\section{References}

1. Uzzaman MM, Jukaku S, Kambal A, Hussain ST. Assessing the long-term outcome of minor lower limb amputations: A 5-year study. Angiology. 2011; 62(5):365-371.

2. Chalya PL, Mabula JB, Dass RM et al. Major limb amputations: A tertiary hospital experience in northwestern Tanzania. J Ortho Surg Research. 2012; $7: 18$.

3. Damme HV, Limet R. Amputations in diabetic patients. Clin Podiatr Med Surg. 2007; 24:569-582.

4. Eskelinen E, Eskelinen A, Alback A, Lepantalo M. Major Amputation incidence decreases both in non-diabetic and in diabetic patients in Helsinki. Scand J Surg. 2006; 95:185-9.

5. Sansam K, Neumann V, O’Connor R, Bhakta B. Predicting walking ability following lower limb amputation: a systematic review of the literature. J Rehabil Med. 2009; 41(8):593-603.

6. Nather A, Wong KL. Distal amputations for the diabetic foot. Diabetic foot \& Ankle. 2013; 4:21288.

7. Yosuf NM, Ahmad AC, Sulong AF et al. Quality of life of diabetes amputees following major and minor lower limb amputations. Med J Malaysia/ 2019; 74(1):25-29.

8. Babic S, Tanaskovic S, Lozuk B et al. Treatment of stump complications after above knee amputation using negative pressure wound therapy. Srp Arh Celok Lek. 2016; 144(9-10):503-6.

9. Jain AKC, Viswanath S. Analysis of Stump Complications Following Major Amputation in Diabetic Foot Complications using Amit Jain's Principle and Practice for Diabetic Foot. Sch J App Med Sci. 2016; 4(3E):986-9.

10. Jain AKC, Viswanath S. Studying major amputation in a developing country using Amit Jain's typing and scoring system for diabetic foot complications-time for standardization of diabetic foot practice. Int Surg J. 2015; 2(1):26-30.

11. Gopal S. Amit Jain's classification for diabetic foot complication: The universal classification supreme. Int J Surg Sci. 2018; 2(2):8-10.

12. Jain AKC, Rajagopalan Gopal S. Testing and Validating Amit Jain's Classification and Scoring System for Diabetic Foot Complications. IJMSIR. 2018; 3(1):22736.

13. Rosner B. In: Fundamentals of Biostatistics, 5th Edition, Duxbury, 2000.

14. Riffenburg RH. In: Statistics in Medicine, 2nd Edition, Academic press, 2005.

15. Rao PSSS, Richard J. In: An Introduction to Biostatistics, A manual for students in health sciences. 4th Ed. New Delhi: Prentice hall of India, 2006.

16. Barawi OAR. Refashioning of amputation stump. Bas $\mathbf{J}$ Surg. 2005; 11(1):116-123.

17. Ngim NE, Ndifon WO, Udosen Am et al. Lower limb amputation in diabetic foot disease: Experience in a tertiary hospital in Southern Nigeria. Afr J Diab Med. 2012; 20(1):13-15.

18. Pickwell K, Siersmo V, Kars M et al. Predictors of lower extremity amputation in patients with an infected diabetic foot ulcer. Diabetes Care. 2015; 38:852-857.

19. Jain AKC, Viswanath S. A study of diabetic foot patients in a tertiary care premiere teaching hospital. Int J Surg Sci. 2019; 2(1):185-187.

20. Jain AKC, Tejasvitaa RS. TO determine the pattern and type of amputation done in diabetic foot patients in a teaching hospital. EAS J Med Sci. 2019; 1(3):94-99.

21. Agha RA, Muneer H, Alqaseer A et al. Major lower limb amputation: Causes, Characteristics and Complications. Bahrain Med Bull. 2017; 39(3):159-161.

22. Vishwakarma N, Rehmani B, Agrawal A, Ray JP. Reamputation rates, morbidity and rehabilitation after lower limb amputations. Int Surg J. 2019; 6(4):12741279.

23. Luka PM, Narsingh NP, Agasty S. A comparative study of lower extremity amputation (LEA) stump outcome in diabetic and non-diabetic patients in a tertiary care hospital of Raipur city, Chattisgarh, India. Int J Res Med Sci. 2013; 3:1488-95.

24. Kumar GK, Souza CD, Diaz EA. Incidence and causes of lower-limb amputations in a tertiary care centre: Evaluation of the medical records in a period of 2 years. Int J Surg Sci. 2018; 2(3):16-19.

25. Salawu ON, Babalola OM, Mejabi JO et al. Major extremity amputations: Indication and post surgery challenges in a Nigeria tertiary institution. Sahel Med J. 2019; 22:8-12. 